\title{
Numerical Simulation of Spot Welding Nugget Formation Process
}

\author{
Xu GAO ${ }^{1, a}$, Xiaohong CHEN ${ }^{1, b}$ and Keqiang $\mathrm{YU}^{1, \mathrm{c}}$ \\ ${ }^{1}$ School of Mechanical Engineering and Automation, University of Science and Technology Liaoning, \\ China \\ agaoxu303@sina.com, ${ }^{\mathrm{b}}$ ascxh@163.com, aakg@@126.com
}

Keywords: dissimilar metals, resistance spot welding, finite difference, numerical simulation

Abstract. Established dissimilar metal iron and nickel resistance spot welding nugget formation temperature field mathematical model for the discretization of the finite difference method, using TFRSW numerical analysis software, data processing module based on MATLAB language, study the dissimilar metal welding nugget formation in the process of resistance spot welding, the size of the develop and change. Put forward the dissimilar conductive metal welding process, the heat transfer effect simulation method and the analysis method of contact resistance. Design the corresponding experiment, verify the correctness of the results of numerical simulation, and analysis in dissimilar metal in the resistance spot welding specification for welding quality are studied.

Resistance spot welding thermal process directly determine the formation and growth of spot welding nugget and the crystallization process, the final decision molten nuclear shape and size. So spot welding thermal process research has a guiding significance to the actual production process analysis.

\section{Spot welding nugget formation thermal process mathematical model}

\section{The establishment of the model}

Spot welding system discretization as shown in figure 1, a total of 1301 nodes, each node are numbered.

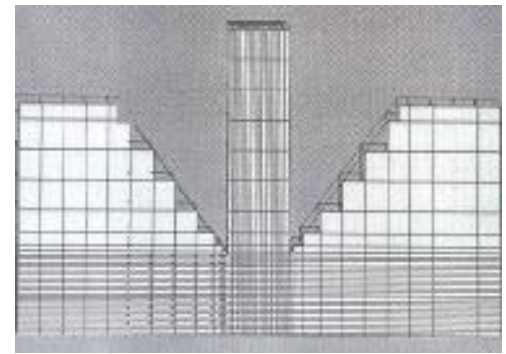

Fig.1 Discretization model of spot welding system

Due to the different area of the spot welding system electrical, thermal, mechanical and performance parameters, such as change is uneven, so using non-uniform grid. Electrode and the workpiece welding area and the area around the grid is populated, and then in turn become sparse.

This model features below ${ }^{[1]}$ : At the same time, considering the whole system voltage field and temperature field and the coupling effect. Resistance heat is a major cause of welding nugget melting, boundary expand. Material coefficient of thermal physical properties with temperature changes in calculated value according to the temperature distribution to each step of the way. Consider the workpiece and the electrode surface by convection and radiation to surrounding heat loss.

Dissimilar materials welding numerical simulation include dissimilar materials thermal physical properties of the nonlinear and boundary nonlinear problem. Solving process and the result is affected 
factors to ignore or simplified: When changing with temperature of thermal physical property parameters for solving the influence of minor or the lack of data, can be simplified processing by interpolation or trial. Electrode pressure effects are ignored.

\section{Electric coupling finite difference method}

Finite difference method is based on the discrete control equations to solve the problem of continuum, according to the figure 1 plan difference grid nodes represent control unit, each node numerical controlled by six nodes around the numerical finite difference equation ${ }^{[2]}$. Temperature and voltage change the larger welding area and the contact area are planned smaller unit grid, because of the influence of contact resistance caused by the voltage distribution and heat yield is regarded as internal resistance of the contact area of the node. The beginning of the simulation from room temperature and calculate the resistivity, density, heat capacity and hardness, calculated by the hardness of the contact resistivity converted to internal resistivity ${ }^{[3]}$. Solve differential equations for potential distribution, node voltage drop, heat production rate and current density. The welding time is divided into several time step, calculating thermal physical property parameters, temperature difference equations for temperature and distribution of all nodes. Cycle the calculation process, different time calculation results are obtained. To solve the huge equations using implicit finite difference iterative method, in order to get the data error is small, the results of convergence. Different calculation parameters influence through the data file coupling between blocks, each calculation results as the next cycle parameter selection based on the temperature.

\section{Control equation difference scheme}

Mainly conduction heat transfer, system heat transfer differential equation for

$$
\frac{\partial}{\partial x}\left(\lambda \frac{\partial T}{\partial x}\right)+\frac{\partial}{\partial y}\left(\lambda \frac{\partial T}{\partial y}\right)+S_{u}+S_{P} T=0
$$

Type of $\lambda$ is the thermal conductivity, $S_{\mathrm{u}}$ is constant and independent of the temperature change, $\mathrm{S}_{\mathrm{p}}$ is with the temperature distribution in the linear change of item.

Temperature field equation in the control volume $\Delta \mathrm{x} \cdot \Delta \mathrm{y}$ integral, finishing available:

$$
a_{p} T_{p}=a_{E} T_{E}+a_{W} T_{W}+a_{N} T_{N}+a_{S} T_{S}+b
$$

Type of: $a_{E}=\lambda_{e} \cdot \Delta y /(\delta x)_{e}, a_{W}=\lambda_{w} \cdot \Delta y /(\delta x)_{w}, a_{N}=\lambda_{n} \cdot \Delta x /(\delta y)_{n}, a_{S}=\lambda_{s} \cdot \Delta x /(\delta y)_{s}, a_{p}=a_{E}+a_{W}+a_{N}+a_{S}-$ $\mathrm{S}_{\mathrm{p}} \cdot \Delta \mathrm{x} \cdot \Delta \mathrm{y}, \quad \mathrm{b}=\mathrm{S}_{\mathrm{u}} \cdot \Delta \mathrm{x} \cdot \Delta \mathrm{y}$

\section{Based on the MATLAB data post-processing}

In TFRSW (Temperature Field of hold the Spot Welding) software, the control volume finite difference method is used, use the control equation for the volume integral to export the discrete equations. Jacobi iteration algorithm is used in large finite difference equations. Data flow in the large capacity hard disk access to complete high-speed electric field and temperature field coupling.

Between FORTRAN and MATLAB for data exchange, data format differences, therefore should be for data format translation, directly through MATLAB processing functions to implement its own matrix.

\section{The establishment of study object model}

In figure 1 model is divided into three parts, including the top and bottom electrodes and central workpiece. Grid represents the model, node represents the temperature field of the location in which the model. Concrete numerical value by special storage nodes DAT file to read. DAT files are generated by FORTRAN program. The storage format is N line single-row type. Their storage way is: A DAT file represents the temperature of each node at a moment, DAT file numerical are regulated by a temperature value of each moment. Including the workpiece and the electrode of each part 
temperature data, mainly choose melting nuclear area. Filtering process is implemented by MATLAB language programming.

\section{Temperature field of the computer simulation results}

The resulting temperature field model is shown in figure 2 .
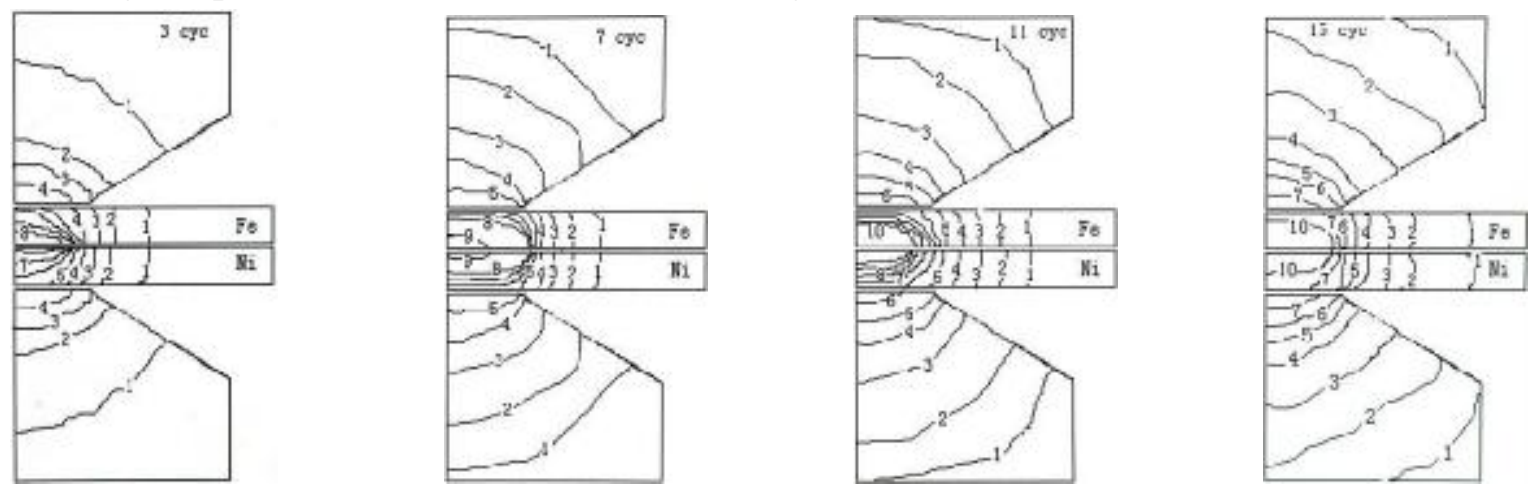

Fig. 2 The temperature field under different heating time

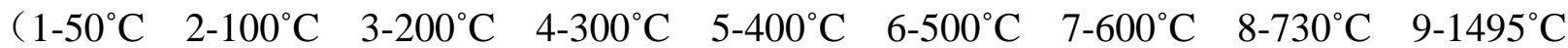 $10-1530^{\circ} \mathrm{C}$ )}

\section{Test analysis}

\section{Test}

Test using the workpiece material is 15 steel and the purity is more than $99.9 \%$ of the nickel, electrode material for copper. Test specification is respectively 8000A, $10000 \mathrm{~A}, 10000 \mathrm{~A}$ and 13000 A, welding time of five cycles to 50 cycles respectively.

\section{Results analysis}

Molten nuclear long axis is located in the joint surface basically, but there are differences between the molten nuclear shapes, the reason is that material thermal physical properties in high temperature difference in a decision. Micrograph of each specification as follows, the white area as the iron, the black area for nickel.

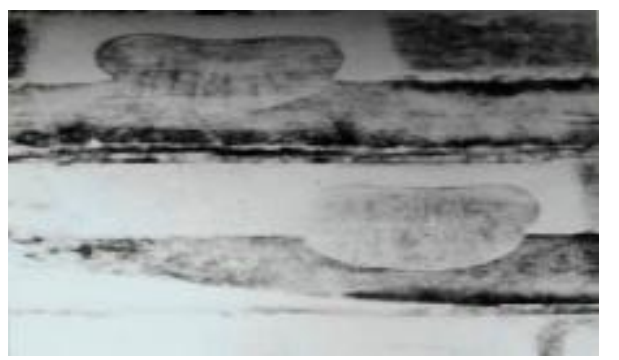

Fig.3 Weld nuggets of 8000 A of 20 cycle (below) and 30 cycle (above)

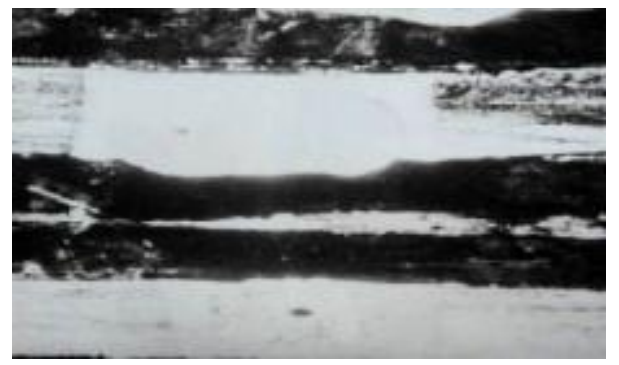

Fig.4 Weld nuggets of 10000 A of 5 cycle (below) and 10 cycle (above) 


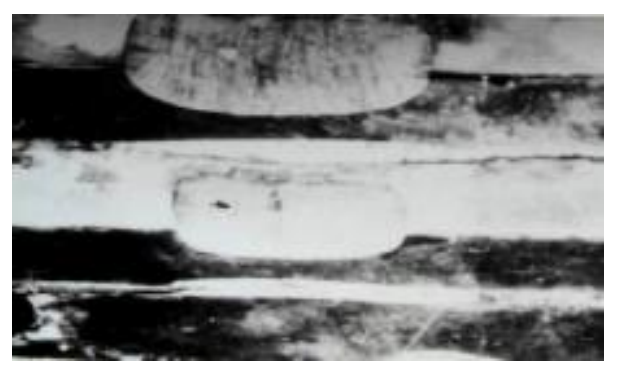

Fig.5 Weld nuggets of 12000 A of 5 cycle (below) and 10 cycle (center) and 15 cycle (above)

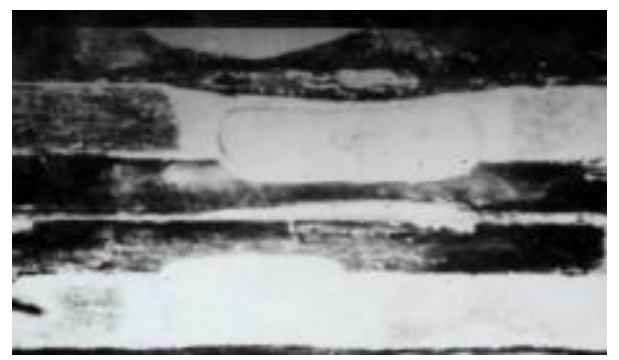

Fig.6 Weld nuggets of 13000 A of 5 cycle (below) and 10 cycle (above)

Along with the welding current is bigger, melt nuclear offset decreased gradually or even disappeared, that's because there are differences between the thermal physical properties of the weldment, strong specification dependence of contact resistance enhancement, heat dissipation difficulties; Specification abate, heat production depends on the internal resistance, heat dissipation, high heat yield and lower the heat capacity of metal melt, priority in molten nuclear to the side.

Specification parameters affecting the formation of molten nuclear are: welding current, welding time and thickness of the workpiece. Current and time effect was similar, can be described as:

(1)In the incubation period without melting phenomenon;

(2)There is a molten nuclear rapid growth period;

(3)Over a period after melting nucleation rate lower;

(4)Molten nuclear disappear.

Current and time parameters affect the difference is that relative size. Under the current specification, melting nucleation incubation period is longer, reduce the period is shorter. Under the time specification, shorter melting nucleation incubation period and rapid growth, reduce the period is longer than the other.

In the above analysis, it was assumed that solder joint can be regarded as a heating element, heat transmission through electrode, electrode and workpiece interface at constant temperature. Solder joint in heat flow as follows:

$$
A \Delta x \rho C_{P} \frac{\Delta T}{\Delta t}=I^{2}-K A \frac{\Delta T}{\Delta x}
$$

In type $\mathrm{A}$ is the electrode surface area, $\rho$ is density, $\mathrm{C}_{\mathrm{P}}$ is the heat capacity, heat conduction coefficient $\mathrm{K}$ is. $\mathrm{R}$ is welding resistance, $I$ is the welding current, $\Delta \mathrm{t}$ is time variable, $\Delta \mathrm{x}$ is weldment thickness, $\Delta \mathrm{T}$ is the temperature variables. The type can be written as:

$$
\Delta T=\frac{I^{2} R}{2 K}\left[\frac{A}{\Delta x}\left(\frac{\Delta x^{2}}{\alpha \Delta t}+1\right)\right]^{-1}
$$

$\alpha$ on behalf of the weldment in type thermal diffusivity, $\Delta \mathrm{T}$ is not affected by melt nuclear size control of this type can explain test results. $\Delta \mathrm{T}$ is proportional to the $I^{2}$, under the current impact, melting nucleation incubation period is longer, under the $\Delta \mathrm{T}$ impact, melting nucleation incubation 
period is shorter.

Correspond to the welding time temperature rise can be described as:

$$
\Delta T \propto\left(\frac{\Delta x}{\alpha \Delta t}+1\right)^{-1}
$$

As in as small $\Delta \mathrm{t}, \Delta \mathrm{T}$ as with $\Delta \mathrm{t}$ is proportional to the, explained the short incubation period and rapid growth. As inside as large $\Delta \mathrm{t}, \Delta \mathrm{x}^{2} / \alpha \Delta \mathrm{t}$ tend to be $0, \Delta \mathrm{T}$ tends to constant value, the same as the $\Delta \mathrm{T}$ value corresponding to the stability of the melt nuclear size of this value can always get in front of the melt nuclear solidification. It accord with the experiment observed in melt nuclear formation of large size molten nuclear began to decrease, gradually disappear.

\section{discuss}

In a current specification, the weld nugget formation experienced several stages: the incubation period, rapid growth, gradually reduce the period and finally disappear. When the welding current is below 8000A, the weld nugget is hard to form, in 8000A 10000A melt nuclear rapid growth of the last for more than 12000A molten nuclear gradually disappear. Welding time effects on the nucleation process is similar to the welding current. First is the incubation period, to more than 5 cycle is melting nucleation. Growth over time, to 10 cycle molten nuclear size increased rapidly, forming stable size. In $10 \sim 15$ cycle, molten nuclear size changes greatly.

In the welding cycle, ring melting nuclear expand outside introversion. Welding current, electrode force and welding time influence the heat flow field in the process of mutual inductance, eventually melt nuclear to change shape ${ }^{[4]}$. When spot welding workpiece with different thickness, welding nuclear in thick pieces of the formation of the more than on thin pieces; When spot welding heterogeneous metal materials, melting most nuclear formation in thermal conductivity with low or high resistance coefficient of the workpieces.

According to the data obtained in the process of extruding cycle stress concentration on the edge of the contact between the electrode and workpiece area and surrounding the workpiece surface ${ }^{[5]}$. Finally in the weld nugget is formed between two workpiece diameter is larger than the electrode diameter.

\section{Conclusion}

(1)Establish heterogeneous metal welding two-dimensional axisymmetric finite difference mathematical analysis model, analyzes the contact resistance and material thermal physical property parameters changes impact on the results of numerical simulation, close to the actual situation.

(2)Using finite difference algorithm, using the TFRSW software, through the MATLAB language to deal effectively with the data, to form the model of the temperature field.

(3)Through the establishment of weld nugget formation temperature field mathematical model, studies the heterogeneous metal welding hot melt nuclear formation, in the process of development and size changes.

(4)Test showed that the model is in good agreement with the actual process, the theory and the calculation result is close to spot welding process. The model and the software can be used to analyze a heterogeneous metal welding molten nuclear formation process.

\section{Reference:}

[1] Ming LEI, Min WANG, Meiqing YIN. A Finite Element Simulation of Spot Welding for Zinc-Coated Steel[J]. Journal of Shanghai Jiaotong University, 2005, 39(7):1033-1037. 
[2] Chunsheng WANG, Yong CHEN, Fengwu HAN, Peide LU, Hongliang WANG. Numerical Simulation for Resistance Spot Welding Nugget Process of Three Layers Stainless Steel Sheets[J]. Chinese Journal of Mechanical Engineering, 2004, 40(1):191-194

[3] Xin LONG, Jianhua WANG, Zengyan ZHANG, Liangwu WEI, Hao LU. Numerical Simulation on The Temperature Distribution in Spot Welding[J]. Journal of Shanghai Jiaotong University, 2001, 35(3):416-419

[4] Fangfang SUN, Mengsheng LI, Yang WANG, Ying ZHAO. Numerical Simulation of Spot Welding for 201 Stainless Steel[J]. Transactions of The China Welding Institution, 2011, 32(2):21-24

[5] Biao CAO, Yihong JIANG, Jianyi WANG. Finite Element Model for Resistance Spot Welding Process[J]. Chinese Journal of Mechanical Engineering, 1995, 31(2):99-104 